\title{
A Research on SDN-based Virtual Fast Transmitting Network Technology
}

\author{
Xu Changbiao ${ }^{1}$, Lu Zhaonan ${ }^{1, a}$ \\ ${ }^{1}$ School of Telecommunication and Information Engineering, \\ Chongqing University of Posts and Telecommunications,Chongqing, China \\ aglenmsen@yahoo.com
}

Keywords: SDN; Network Virtualization; Delay; Packet Loss Rate;

\begin{abstract}
This paper presents a novel chain-like fast transmitting network which is based on the Software Defined Networking (SDN) virtualization technology to deal with the problems that the current mechanism of reducing packets delay is complicated and there is quite a high Processing delay during matching flowtable in some routers. In this design, different data will be mapped to different virtual transport network and latency-sensitive data will be handled by the fast transport mechanism without matching complicated flowtable. The simulation show that our design could effectively improve the transport efficiency of the network without affecting the normal data.
\end{abstract}

\section{INTRODUCTION}

With the high speed of development of the Internet technology and network service, novel network applications spring up (e.g. VoIP、VOD and Video Conference etc. ). the common issue of these new applications is that they have a higher requirement on quality of service(QoS), especially on delay and jitter. However,the current transport network which is based on the Best Effort Service could not provide the corresponding QoS for these applications. To address this problem,network operators introduce Differentiated Services(DiffServ) and Integrated Services(IntServ) ${ }^{[1]}$. DiffServ is a protocol for specifying and controlling network traffic by using class of service that marks certain precedence to a packet or flow,which is widely used on VPN and VoIP services. In the process of data transmitting, a router would use different Per-Hob Behavior(PHB) according to the different COS value. This kind of approach could provide some QoS guarantees for some packets. However, the transmitting model of PHB-based could not provide fast transport for the delay-sensitive packets when it suffer from partial congest result from normal data;Integrated Services(IntServ) could provide fine-grained class for various data which is used for multimedia in the early time.It used REVP,a signaling protocol to achieve the reserve of resources.Although this guaranteed service offers bounded Delay and Packet Loss for the required applications, it still difficult to deploy IntServ into Large-scale backbone network because it requires a complex signaling protocol such as RSVP to reserve resources and much signals will occupy quite a number of bandwidth or other network resource.Besides,the transmitting model of reserving resource is not qualify to the burstiness and volatility of IP data.

In order to provide proper transport quality to different traffic, virtual networks attract many attention from industries and academic world. Through some virtualization technologies, several virtual networks which are isolated with others logically could operate on the same physical network an the same time. The traffic and manage of different virtual network are isolated without interference. Therefor different virtual networks could use different protocols to execute different functions. In addition, this kind of technologies could also improve the utilization ratio and reduce the costs of management and maintenance of a network. In this paper,we use SDN (Software Defined Networking) ${ }^{[2]}$ virtualization technologies and the uncoupling mechanism of control plane and data plane in routing and switching gear to establish 3 virtual networks on the same physical network. The 3 networks includes of a Fast Transmitting Network、 a Low Packet Loss Network and a normal Best Effort Network.. The simulation result show that our design could effectively 
improve the transport efficiency of the network without affecting the normal data.

\section{SDN Virtual Networks}

In Fig.1,during the process of the network operation, the SDN router will report the current work situations (e.g.matching rate of the flow entry flow load、 packet loss delay etc.)to the relative SDN controller termly. According to these corresponding information,the controller would evaluate the current operation situation of this SDN network and sending flow tables to relative SDN routers ${ }^{[3]}$. Because the controller could decide routers' behaviors by updating flow tables, it is easy to establish several virtual networks on the same same physical topology. In this SDN network architecture,the Best Effort Network data would be routed by matching normal flow tables which are created by Dijkstra's Algorithm and the weights includes load of a router and the link bandwidth.

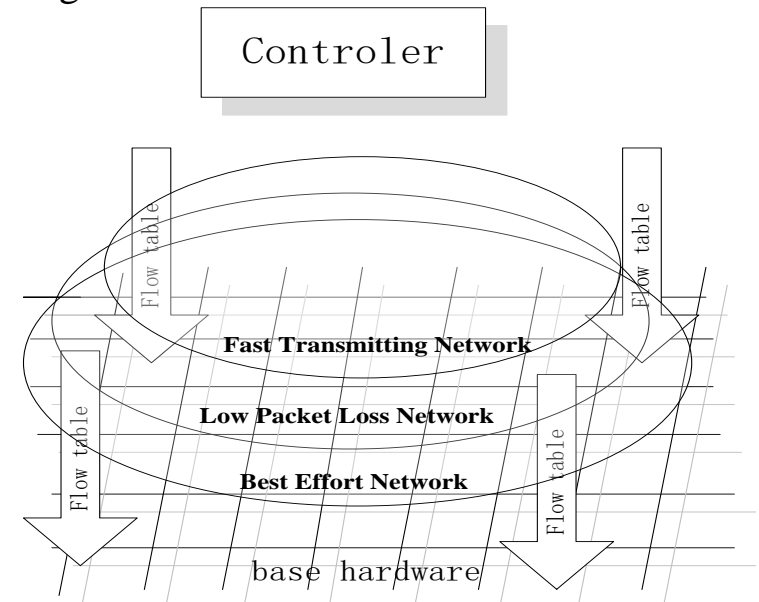

Fig.1.Overview of SDN Virtual Networks architecture

\subsection{Fast transmitting network}

It is different with the process of handling normal packets,shown as Fig.2, the number and matching content of flow entries for a delay-sensitive data in the Fast Transmitting Network are simplified greatly.

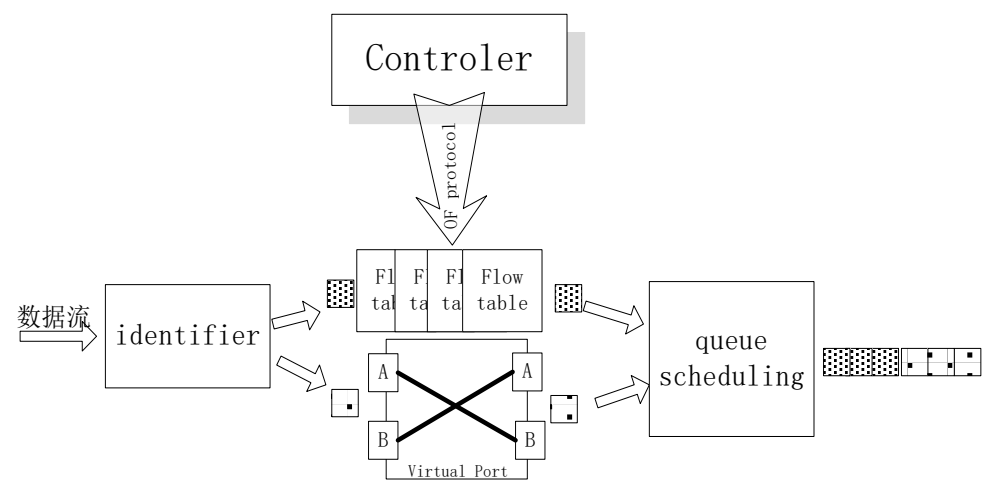

Fig.2 Transmitting Mechanism for Fast Network

In this way, the time that searching and matching some flow entries in one router is shortened,which maximally reduces nodal processing delay of a packet during it is transmitting in this network.In addition,under the premise of load balancing, we introduced Resource preemption and strict priority queue scheduling mechanism to provide more bandwidth resources for delay-sensitive packets.According to the global view generated by SDN controller and minimum connected dominating set algorithm ${ }^{[4]}$, we design a chain-like fast transmitting network and relative core SDN routers.because the core nodes contain dominating set nodes,all the normal nodes could access fast network through one hop. The biggest advantage of a chain-like network is that there are only two couples of output port and input port and these ports are stationary over a period of time. Based on above features,the flow entries only need to include priority and hop number. There is a 
little modification required to OpenFlow ${ }^{[5]}$ which is to increase the size of the tuple used for flow identification.Tab.1 shows the 12 tuple. When a core router received a delay-sensitive packet, the identifier module will check the tab field and the count field would be minuend 1 then transported the packet to the corresponding output port.if the count field is " 0 ",the relative core router would handle it as a normal packets and send it to the Best Effort Network.

Tab.1 OpenFlow Twelve-tuple for Fast Transmitting Network rules

\begin{tabular}{|c|c|c|c|c|c|c|c|c|c|c|}
\hline In & VLAN & & Ethern & & Virtua & twork & & IP & & Transport \\
\hline Port & ID & SA & DA & Type & Tab & Count & SA & DA & Proto & \\
\hline
\end{tabular}

\subsection{Low Packet Loss Network}

In order to provide appropriate network resource for the delay-insensitive but Packet Loss-sensitive packets,we defined Low Packet Loss Network. Since the delay-sensitive packets have the highest priority, this kind of data would occupy much of bandwidth resources from the other data.Hence, the SDN controller should generate particular flow tables to prevent overmuch overlap between the Low Packet Loss Network and the Virtual Fast Transmitting Networks.In addition,SDN router would keep a longer queue size and a higher priority than the normal data in the relative output port.Moreover, when the packet loss rate exceeds a threshold,SDN router would feedback this information to the controller and ask for updating new flow entry.

\section{Simulation and Conclusions}

We used OPNET 14.5 to establish a simple SDN simulation platform.In this simulation,the SDN controller generates different kinds of flow tables to different routers- the normal packets are transmitted by Dijkstra's Algorithm and delay-sensitive packets and Loss-sensitive packets are handled by corresponding transport mechanism respectively. The data sources generate different packets randomly and mark relative tab on these packets. The link bandwidth is $10 \mathrm{M} / \mathrm{s}$, the queue size of delay-sensitive packets L Loss-sensitive packets and normal packets are 500/5000/10000 respectively. And the router handle every packets with a constant rate $5 \mathrm{P} / \mathrm{s}$. The result are shown in Fig.3 and in Fig.4

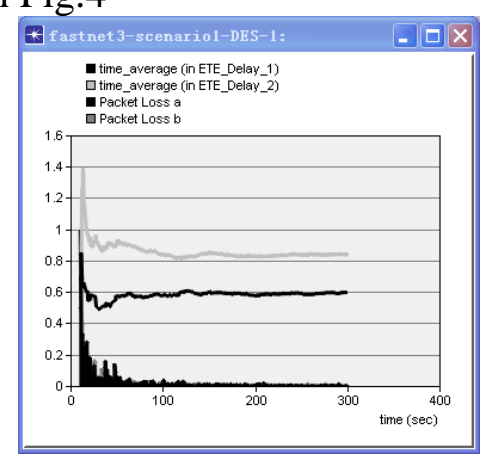

Fig.3 delay result in Virtual Network

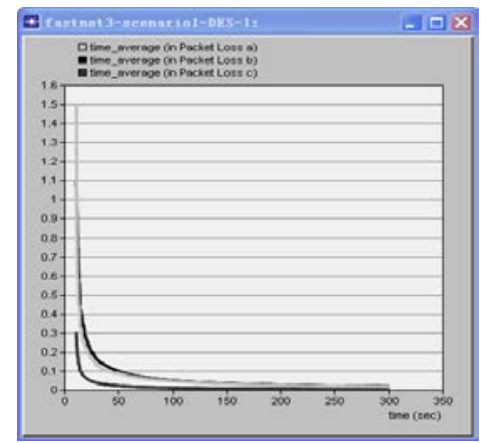

Fig.4 Loss Rate result in Virtual Network

In Fig.3, delay-sensitive packets are transmitted on the Fast transmitting network,because the process time is shortened and the transport link is simplified,the transmitting delay is lower than the normal data.besides,changeless transmission route reduces data jitter to some extent. In Fig.4, due to SDN router maintain a bigger queue size and during the process of transmitting data,the loss-sensitive packets could avoid Fast transmitting network, Low Loss Network could transport data with a lower packet loss rate.

SDN(software defined network) separates logical plane and data plane form a single box, controller provides global view to the user and brings more possibles to the routing rule for routers. This design inspired by the way of train operation,normal data is like the normal passengers using the slow network hop-by-hop until to arrive the destination.But the delay-sensitive data is like senior passengers using bullet trains to achieve the destination directly without worrying about transfer.now,our design could achieve a coarse-grained QoS for some special packets,in the further research,our design will provide fine-grained QoS service to different applications(e.g. Voice、video 
streaming signaling .etc.) and to improve the utilization rate of network resource.

\section{REFERENCES}

[1]Lee T. Multiprotocol laber switching (MPLS) and differentiated services (DS) as quality of service (QoS) solutions[C]//Advanced Communication Technology, 2005, ICACT 2005. The 7th International Conference on. IEEE, 2005, 2: 1039-1043.

[2]McKeown N, Anderson T, Balakrishnan $\mathrm{H}$, et al. OpenFlow: enabling innovation in campus networks[J]. ACM SIGCOMM Computer Communication Review, 2008, 38(2): 69-74.

[3]Luo X, Ma S, Jin Y. HADES: A compatible SDN based network virtualization architecture[C]//Optical Internet 2014 (COIN), 2014 12th International Conference on. IEEE, 2014: $1-2$.

[4] Jing Z, Chun-Fu J. Minimum Connected Dominating Set Algorithm with Weight in Ad Hoc network[C] //Wireless Communications, Networking and Mobile Computing, 2008. WiCOM'08. 4th International Conference on. IEEE, 2008: 1-4.

[5]“Open Flow 1.4.0 Specification ,” Apr. 2014. [ Online ] . Available : htps : // www . open networking .org / images / stories / downloads / sdn-resources /onf - specifications /openflow / openflow -spec -v1.4.0.pdf 